\title{
Living donor liver transplantation in South Africa: the donor experience
}

\author{
J Botha, ${ }^{1}$ B Ströbele, ${ }^{1}$ J Loveland, ${ }^{1}$ S Rambarran, ${ }^{1}$ R Britz, ${ }^{1}$ H Etheredge, ${ }^{2} \mathrm{H}$ Maher, ${ }^{2}$ S Bolon, ${ }^{4} \mathrm{M}$ Eagar, ${ }^{3}$ A Beeton, ${ }^{3}$ \\ L Brannigan, ${ }^{3} \mathrm{R}$ Mononyane, ${ }^{3} \mathrm{~S}$ Bannan, ${ }^{3} \mathrm{M}$ Morford, ${ }^{3} \mathrm{O}$ Smith, ${ }^{4} \mathrm{~J}_{\text {Fabian }}{ }^{2}$ \\ ${ }^{1}$ Wits Donald Gordon Medical Centre, Department of Surgery, School of Clinical Medicine, Faculty of Health Sciences, \\ University of the Witwatersrand, South Africa \\ ${ }^{2}$ Wits Donald Gordon Medical Centre and Department of Internal Medicine, Faculty of Health Sciences, University of the \\ Witwatersrand, Johannesburg, South Africa \\ ${ }^{3}$ Wits Donald Gordon Medical Centre Transplant Anaesthesia, School of Clinical Medicine, Faculty of Health Sciences, \\ University of the Witwatersrand, South Africa \\ ${ }^{4}$ Department of Anaesthesia and Critical Care, Charlotte Maxeke Johannesburg Academic Hospital, University of the \\ Witwatersrand, South Africa
}

Corresponding author: Dr Jean Botha (jeanf.botha@gmail.com)

\begin{abstract}
Background: Living donor liver transplantation (LDLT) plays a crucial role in liver transplant programmes, particularly in regions with a scarcity of deceased donor organs and especially for paediatric recipients. LDLT is a complex and demanding procedure which places a healthy living donor in harm's way. Donor safety is therefore the overriding concern. This study aimed to report our standardised approach to the evaluation, technical aspects and outcomes of LDLT donor hepatectomy at Wits Donald Gordon Medical Centre.

Methods: The study population consisted of all patients undergoing LDLT donor hepatectomy since the inception of the programme in March 2013 until 2018. Sixty five living donor hepatectomies were performed. Primary outcome measures included donor demographics, operative time, peak bilirubin, aspartate and alanine transaminase levels postoperatively, length of hospital stay and postoperative complications using the Clavien-Dindo classification.

Results: The majority of the donors were female, most were parents with mothers being the donor almost $85 \%$ of the time. The median operative time was 374 minutes with a downward trend over time as experience was gained. The median length of hospital stay was 7 days. There was no mortality and the complication rate was $30 \%$ with the majority being minor (Grade 1).

Conclusion: Living donor liver transplant from adult-to-child has been successfully initiated in South Africa. Living donor hepatectomy can be safely performed with acceptable outcomes for the donor. Wait-list mortality however remains unacceptably high. Expansion of LDLT as well as real change in deceased donor policy is required to address this issue.
\end{abstract}

S Afr J Surg 2019;57(3)

http://dx.doi.org/10.17159/2078-5151/2019/v57n3a2998

\section{Introduction}

Liver transplantation is the standard of care for children with decompensated end-stage liver disease. Paediatric liver transplantation was initiated at Wits Donald Gordon Medical Centre (WDGMC) in Johannesburg, South Africa in 2005; initially utilising deceased donor reduced size and whole grafts. However internationally, living donor liver transplantation(LDLT) has emerged as an important alternative for children with end-stage liver disease due to the limited availability of deceased donor organs. WDGMC is the only paediatric liver transplant programme in sub-Saharan Africa offering living donor liver transplantation, indicating the shortage of this form of medical therapy in the region. ${ }^{1,2}$ The deceased donor shortage in South Africa mirrors that occurring throughout the world and led to the emergence and sustained growth of split-liver and LDLT at our centre. LDLT was introduced at WDGMC in 2013 and has become a major mode of paediatric liver transplantation, now accounting for $50 \%$ of the liver allografts transplanted into children at our institution. In this, the first report of living donor outcomes from Southern Africa, we describe our approach to evaluation of potential candidates, surgical technique and outcomes of living donor left lateral segment (LLS) hepatectomy from 2013 to 2018.

\section{Materials and Methods}

We conducted a review of donors undergoing adult-topaediatric living liver donation at our institution. Between 
Table 1. Pre-operative assessment of the potential living liver donor.

\begin{tabular}{ll}
\hline Standard Living Donor Workup \\
\hline Screening & Relationship to patient \\
& Blood type \\
& Height, weight and BMI \\
& Medical, surgical and family history \\
& Alcohol, smoking and drug history \\
Laboratory Tests & Full blood count and platelets \\
& Urea and electrolytes, glucose, lipid profile \\
& Liver function tests \\
& TPHA, HIV, HAV, HCV, HBV \\
& CMV and EBV serology \\
ECG and Echo cardiogram \\
Respiratory & $\begin{array}{l}\text { Chest X-ray, Spirometry and Arterial blood } \\
\text { gas }\end{array}$ \\
Liver & Ultrasound abdomen \\
& Triple phase CT scan - vascular anatomy \\
Consultations & $\begin{array}{l}\text { Hepatology, transplant surgery, nurse } \\
\text { co-ordinator, critical care and psychology }\end{array}$
\end{tabular}

March 2013 and December 2018 we performed 65 living donor hepatectomies at WDGMC. This study was approved by the Human Research Ethics Committee (Medical) of the University of the Witwatersrand (No. M160367). Living donation is always presented to the recipient family as an option for achieving transplantation. Potential living donors are not actively pursued in an attempt to avoid coercion. Donors were selected according to a standardised protocol which started with a medical and social history questionnaire. If approved, potential donors underwent a full evaluation involving laboratory and radiological investigations and numerous consultations with surgeons, hepatologists, critical care specialists and mental health care professionals as part of the evaluation of their candidacy to undergo living donor hepatectomy (Table 1).

Research data collected included donor demographics, relationship to recipient, CT scan predicted graft volume, total operative time, blood loss, actual graft weight, graft weight to recipient weight ratio (GWRW), length of hospital stay, peak postoperative bilirubin levels, aspartate aminotransferase (AST) and alanine aminotransferase (ALT), and postoperative complications according to the Clavien-Dindo scoring system. $^{3}$

The upper age limit that was permitted was 50 years and a maximum body mass index $(\mathrm{BMI}) \leq 30$. The vascular anatomy of the potential graft was assessed by contrast-enhanced multi-phase high resolution CT scan (Figure 1). Volumetric analysis of the intended graft is performed using the "liver segmentation" add-on to our Philips Intellispace ${ }^{\circledR}$ radiology software suite in order to assess the total liver volume, graft volume and residual liver volume (Figure 2). Preoperative assessment of biliary anatomy was not routinely performed

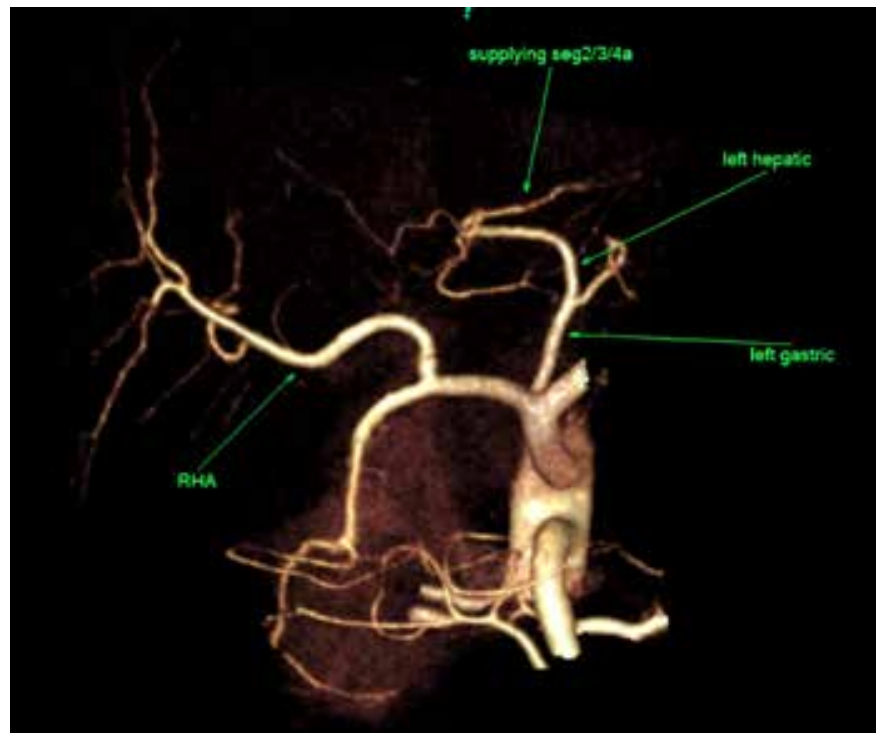

Figure 1. Three-dimensional reconstruction of a quadruple phase CT scan demonstrating the hepatic arterial anatomy. In this case a large replaced left hepatic artery is seen arising from the left gastric artery.

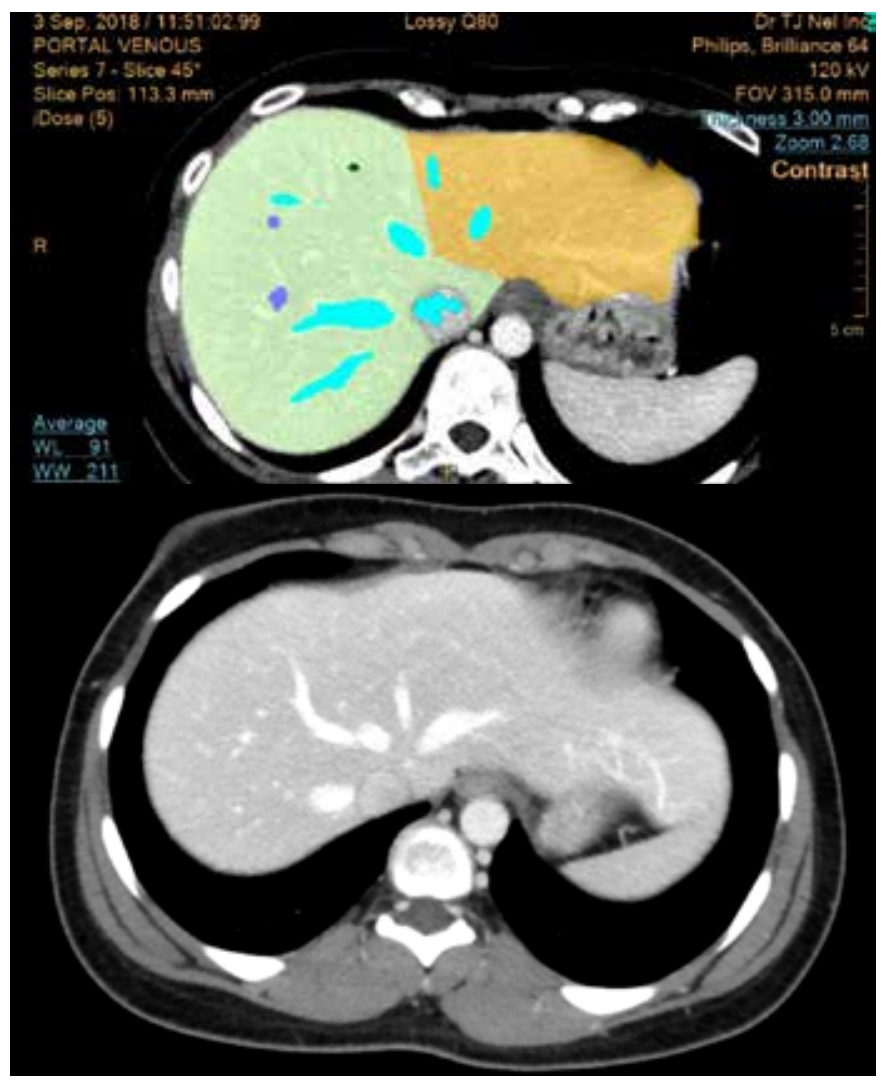

Figure 2. The delayed phase of the contrast enhanced CT scan clearly demonstrating the hepatic venous anatomy. From these images, a 'virtual' transection plane between the left and middle hepatic veins is constructed and subsequent volumetric assessment of the LLS and the remnant liver can be determined.

and on-table cholangiography was utilised for determination of the site of division of the left hepatic duct. We do not 
routinely perform liver biopsies as part of the evaluation, but livers that are deemed steatotic on imaging are further evaluated with a biopsy and histopathology evaluation. We do not proceed with donation if macrosteatosis exceeds $10 \%$.

Initially only blood group compatible donor-recipient pairs were used but more recently we have begun to use ABO incompatible (ABOi) grafts, particularly for children with acute liver failure, with a protocol of plasma exchange and rituximab in the perioperative period. Additionally, we do not exclude donors who are hepatitis B core antibody $(\mathrm{HBcAb})$ positive, and naïve recipients of $\mathrm{HBcAb}$ positive livers are treated prophylactically with lamivudine to prevent transmission of hepatitis B virus (HBV). Females $<6$ months postpartum were excluded from donation as well as patients on oral contraceptives for less than 6 weeks. For certain inborn errors of metabolism, liver transplantation represents the best available therapeutic approach. In this situation a living donor from the parent may be a heterozygous carrier of the disease and may need additional screening to ensure the success of the procedure both for the donor as well as the recipient. ${ }^{4,5}$

\section{Operative Technique}

As all of the hepatectomies were LLS grafts, the following description is of the LLS operation. After anaesthetic induction, central and arterial lines are placed for all donors, as are sequential compression devices on both calves. Epidural catheters are routinely used for pain management in order to limit the use of systemic narcotic analgesia and subsequent opioid induced ileus. Prophylactic antibiotics are administered and continued for 24 hours postoperatively, cefazolin being the drug of choice. Low molecular weight heparin is commenced after the surgery.

Donors are positioned supine with the right arm abducted to $90^{\circ}$ with particular attention being paid to the prevention of brachial plexus injury. During the early part of the series, a bilateral subcostal incision was used but this was changed to a vertical upper midline incision from the xyphoid to just below the umbilicus early in the series. A Thompson table-mounted retractor is placed and affords excellent exposure of the liver and porta hepatis.

After division of the falciform and triangular ligaments, the gastrohepatic omentum is divided exposing the caudate lobe, followed by dissection of the porta hepatis where the left hepatic artery is isolated. The left hepatic artery is dissected along its course and often an artery to segment IV is encountered, which may be divided here, or in the parenchyma depending on exposure required. The left portal vein is dissected and caudate lobe branches are ligated and divided. The left hepatic duct is identified and the potential site for transection is marked with a metal clip. A cholangiogram is then performed via the cystic duct and the site of division of the duct confirmed insuring safety for both the donor and the recipient (Figure 3). The gallbladder is then removed and the cystic duct ligated.

An intraoperative ultrasound is then performed in order to identify and mark the course of the middle hepatic vein.

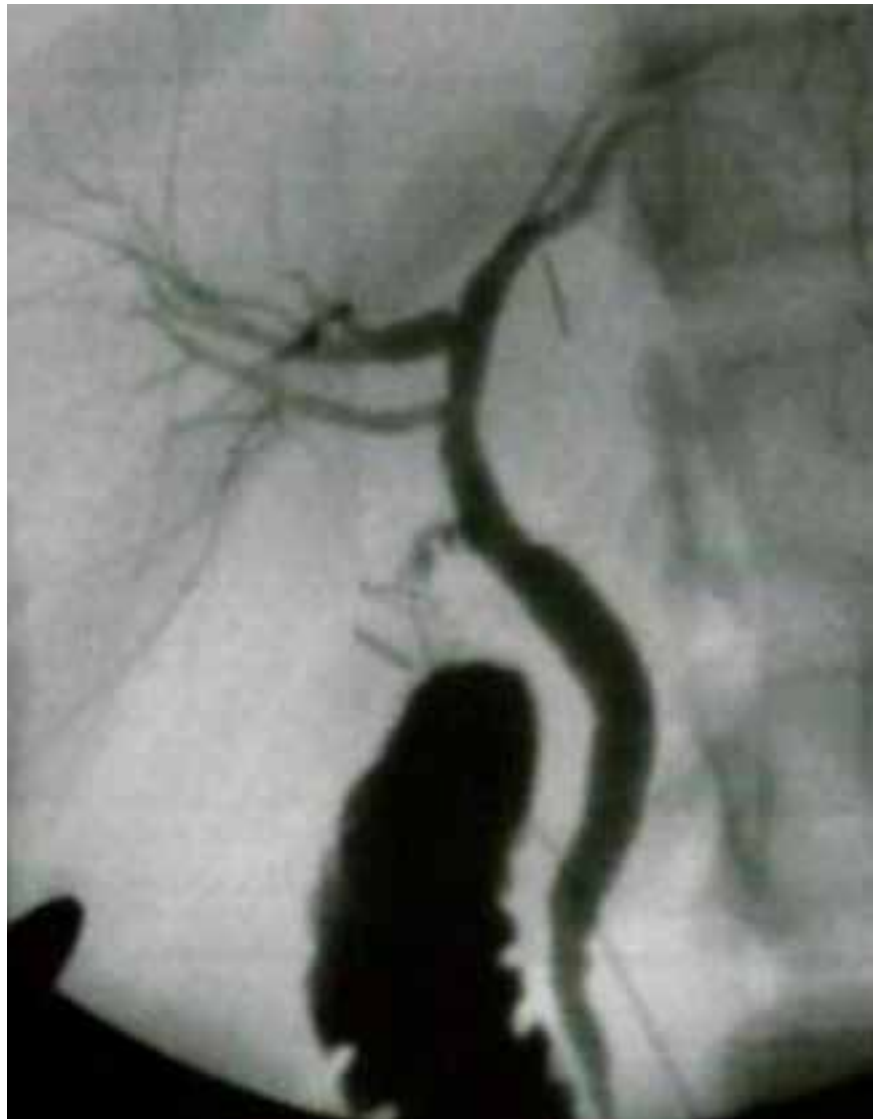

Figure 3. Intra-operative cholangiogram performed via cannulation of the cystic duct. A small metal clip delineates the division of the left hepatic duct into segment II and III hepatic ducts. Transection of the duct will be distal to the metal clip towards the common bile duct bifurcation. Also seen is the aberrant anatomy of the right hepatic duct system.

Liver transection is performed with an ultrasonic aspirator (Cavitron Ultrasonic Surgical Aspirator (CUSA), Valleylab Corp., Boulder Colorado) as well as the Ligasure Precise (Medtronic, Minneapolis, Minnesota). The left hepatic duct is sharply divided during the parenchymal transection so as to minimise devascularisation of the duct. The transection proceeds superiorly towards the left hepatic vein until the only remaining attachments of the left lateral segment are the left hepatic artery, left portal vein and left hepatic vein. After administration of intravenous heparin, the left hepatic artery is ligated close to its origin and divided with a scalpel. A vascular clamp is placed across the origin of the left portal vein and then divided sharply distal to the clamp. The left lateral segment is then retracted inferiorly and a vascular clamp placed across the junction of the left and middle hepatic vein slightly encroaching onto the middle hepatic vein. The left hepatic vein is divided on the graft side of the clamp. The graft is removed and flushed on the back-table with cold preservation solution through the portal vein. Using a syringe and a cannula, the left hepatic artery is also flushed with cold preservation solution.

In the donor, the left hepatic vein orifice is oversewn with a 4/0 Polypropylene suture in a running continuous fashion, 


\section{Relationship to Recipient}

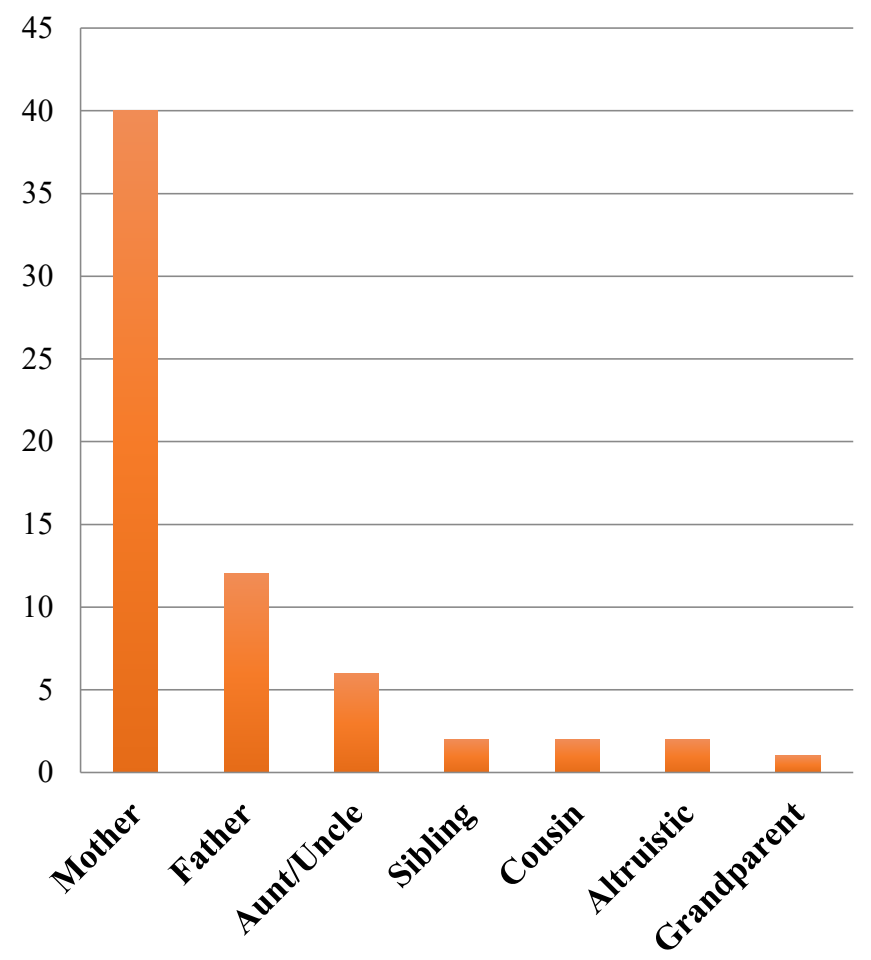

Figure 4. Donor and recipient relationship.

similarly the left portal vein orifice is oversewn with a $6 / 0$ Polypropylene suture. The open end of the left hepatic duct is closed with a $6 / 0$ running absorbable suture. The cut surface of the liver is carefully examined for bleeding and bile leaks and these are repaired with interrupted sutures. On the backtable the graft is inspected to insure adequate length of portal vein, it is weighed and packaged for transport to the recipient operating room.
Table 2. Post-operative complications graded according to the Clavien-Dindo scoring system.

\begin{tabular}{|c|c|c|}
\hline \multicolumn{3}{|l|}{ Post Operative Morbidity } \\
\hline & Number & $\%$ \\
\hline Total no. of complications & 20 & 30.7 \\
\hline \multicolumn{3}{|l|}{ Clavien Grade } \\
\hline I & 11 & 55 \\
\hline II & 2 & 10 \\
\hline IIIa & 2 & 10 \\
\hline IIIb & 3 & 15 \\
\hline IV & 2 & 10 \\
\hline $\mathrm{V}$ & 0 & 0 \\
\hline \multicolumn{3}{|l|}{ Grade IV Complications } \\
\hline \multicolumn{3}{|c|}{$\begin{array}{l}\text { Bowel perforation with multiple laparotomies, TPN, abdomina } \\
\text { wall reconstruction }\end{array}$} \\
\hline \multicolumn{3}{|c|}{ Respiratory arrest due to inadvertent opioid overdose } \\
\hline
\end{tabular}

\section{Results}

During the study period, a total of 65 LDLT hepatectomies were performed at our institution. Of the 65 donors, 50 were female and 15 were male. The most common relationship to the recipient was a parent $(50 / 65)$ with almost $85 \%$ of the parents being the mother. Two were siblings, 6 were either an aunt or uncle, 4 were cousins, 1 grandparent and there were 2 altruistic donors (Figure 4). All 65 patients underwent left lateral segmentectomies (segments II and III). The mean age of the patients was 31 years ( $\mathrm{SD} \pm 6.8$ years), the median and interquartile range for weight, was $66.2 \mathrm{~kg}(58.8-75 \mathrm{~kg})$, for height 1.66 metres (1.58-1.71), and for BMI 24 (22-26) respectively.

Of the perioperative characteristics, the median (IQR) operative time (wheels in to wheels out) was 347 minutes

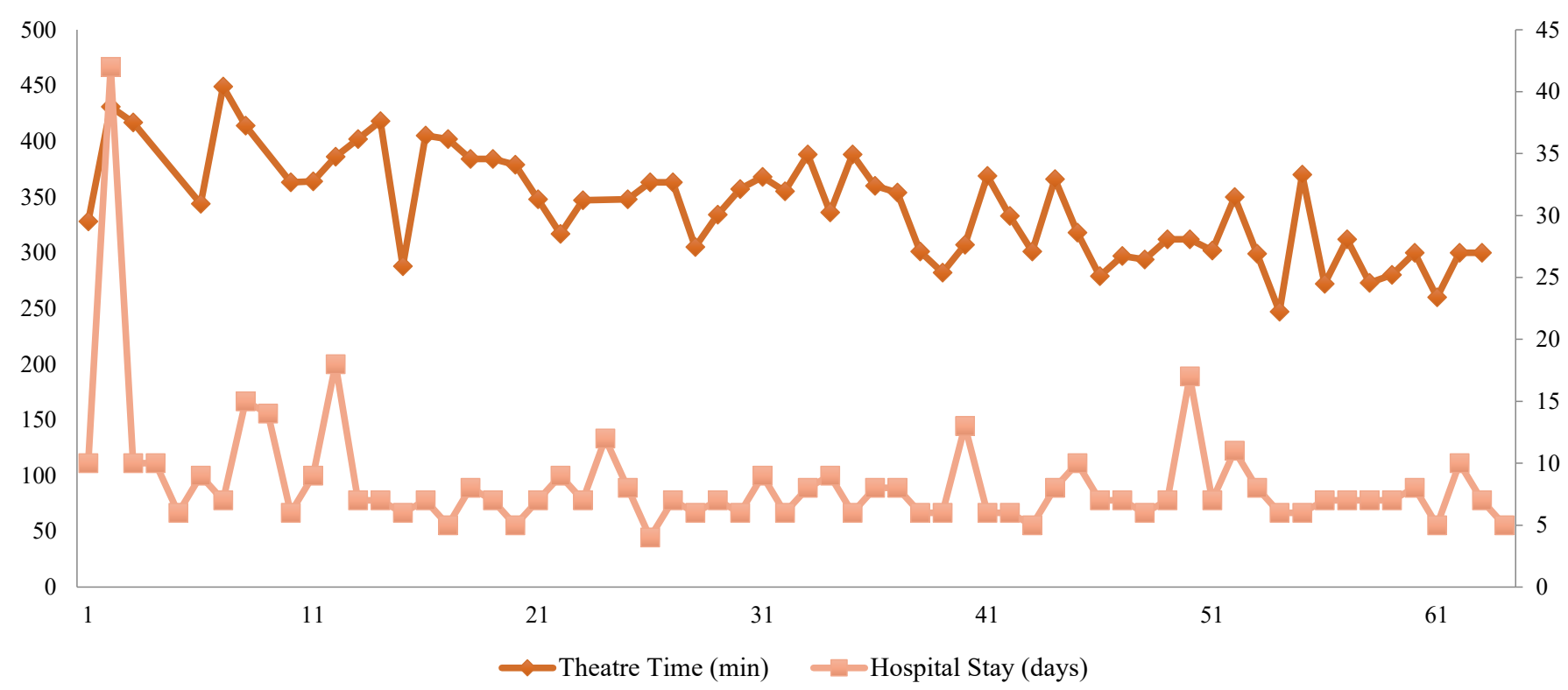

Figure 5. Hospital stay (days) on the right axis and operative time (minutes) left axis, the latter demonstrating a trend towards shorter operative times as the learning curve progressed. 
(301-378 $\mathrm{min}$ ) and appeared to decrease over time as more experience was gained by the entire operative team (Figure 5). Blood loss was minimal and no patient in the series required blood transfusion. The median (IQR) length of hospital stay was 7 days (6-9 days). Minimal disturbance in liver function occurred as demonstrated by the median peak in bilirubin (22mmol/l, IQR 16-31), AST (200 IU/l, QR 126-335) and ALT (232 IU/l, IQR 123-448) levels.

The postoperative complications according to the Clavien system are presented in detail in Table 2. Importantly, there has been no mortality in any of the cases and no grade $\mathrm{V}$ complications. There were 2 grade IV complications, one was a respiratory arrest after a medication error where a patient received an overdose of intravenous opiate. She was successfully resuscitated with no long-term sequelae. The other grade IV complication deserves special mention: The second case in our series was a 32 year old female donating to her child with biliary atresia. The operation proceeded uneventfully; a LLS graft was procured and successfully transplanted into the child who made a good recovery after transplant. On postoperative days 4 and 5 the donor developed worsening abdominal pain and nausea. A CT scan demonstrated an increased amount of free fluid in the peritoneal cavity and a small amount of free air, and the patient was subsequently taken to the operating room. At laparotomy a jejunal perforation was found with marked contamination of the abdominal cavity. The perforation was debrided and primarily repaired with interrupted 4/0 absorbable monofilament suture and the peritoneal cavity washed out.

Four days after re-look laparotomy she again had worsening abdominal pain and was taken back to the operating room, where the perforation was found to have reopened and marked peritonitis and contamination were found. She was systemically septic and at this point we elected to bring out a high loop jejunostomy, left her sheath open and closed the skin. The patient recovered in ICU without further need for surgery and was discharged from hospital on postoperative day 42 and managed on home TPN for a further 6 months. She returned for take-down of the stoma and repair of her abdominal wall with a mesh. She recovered from this operation and 3 years later carried a pregnancy to full term and delivered a healthy baby. She remains well with no recurrent hernia 5 years after the living donor hepatectomy.

In addition to the two grade IV complications, there were 3 biliary complications all being cut surface leaks that responded to drainage with 1 of the patients requiring an endoscopically placed stent. One patient returned to the operating room 24 hours after surgery for bleeding from the cut surface of the liver.

\section{Discussion}

Living donor liver transplantation offers a much needed source of donor livers for paediatric liver transplant recipients in Southern Africa, a geographic region where organ transplantation is constrained by a very complex interaction of factors at all levels of the South African health care system leading to a critical shortage of deceased donor organs. ${ }^{6} \mathrm{~A}$ collaboration between our centre and the transplant program at the University of Nebraska Medical Center facilitated the initiation and development of LDLT in Johannesburg. This is the first report of LDLT from South Africa, a region with a heavy burden of liver disease, but where the provision of a resource intense form of therapy such as liver transplantation is complicated by low organ donor rates as well as resource shortages and logistical limitations.

Since the introduction of LDLT, the safety of the donor has always been the overriding concern. Yet, despite efforts to improve donor safety, living donor deaths do occur and this risk has been estimated to be between $0.02-0.15 \%{ }^{7}$ The age cut-off of 50 years or younger that we imposed for potential donors is admittedly somewhat stringent and a more reasonable approach going forward would be to increase the age limit and rather focus on a potential donor's physiologic condition. Importantly our donor survival is $100 \%$ and the complication rate is $30.7 \%$, of which the majority were minor complications (65\%, CD I and II). Our overall complication rate is acceptable and similar to that reported internationally. The rate of major complications $(\geq$ Grade 3 ) in this South African experience was $7 \%(5 / 65)$ and is well within the reported range from studies in Europe and Asia of 2-12\%. ${ }^{8,9,10}$ While hepatectomy inherently carries the risk of blood loss, none of our donors required blood transfusion during the surgery and as a result we have ceased the use of a cell saving device during this operation.

The recently described LDLT experiences from Brazil and India are very encouraging. ${ }^{11,12}$ These middle-income countries, similar to South Africa, have been able to expand the delivery of this type of advanced health care despite the socio-economic challenges mentioned above. Brazil has seen a sharp rise in the number of liver transplants which has been attributed partly to an increase in the use of split liver transplants. Split liver transplantation was introduced at our centre in order to improve the access of our paediatric recipients to organs. Currently about a quarter of our children receive a cadaveric split liver allograft, fifty percent receive a LDLT and the remainder receive whole grafts. Despite this we still have a waiting list mortality of approximately $20 \%$ in our paediatric liver transplant population. The ongoing need for, and further expansion of both LDLT and deceased donor transplantation in South Africa is therefore very important.

Apart from the previously mentioned socio-economic challenges, there are other special problems facing transplant programmes in Africa. The burden of HIV is one such issue. Until recently, HIV had been an exclusion criteria for being a living liver donor. However, we are not infrequently faced with the situation of healthy young adults living with wellcontrolled HIV who have HIV-negative children that present to our centre with children in either acute or chronic liver failure. It is these parents who have challenged us as to why being HIV-positive excludes them from being living donors and saving the lives of their children. This unique situation has led to the first successful LDLT from an HIV-positive mother 
to her HIV-negative child at our institution. ${ }^{13}$ Going forward, the success of this case has resulted in formalizing this as a research protocol within our programme. It is our hope that the ability to offer LDLT to families where the donor is HIVpositive will open up new possibilities for LDLT, particularly in this situation where potential donors were previously denied an opportunity to save the life of their child.

Female donors were the most common in our series, mostly due to women being relatively smaller than men and considering the fact that the mean weight of our recipients is just under $10 \mathrm{~kg}$. We share the experience of others that females tend to have what is sometimes referred to as a 'flat fish' shape to the left lobe as opposed to the 'puffy fish' or 'box' shape that males tend to have. ${ }^{14}$ This also means that we have not yet encountered the need for hyper-reduced grafts. There have been 2 altruistic donors in this series, this necessitates that we seek approval from the Minister of Health through the Medical Advisory Committee (MAC) for organ transplantation. The extra administrative work can lengthen the process to transplantation, however when urgent approval is required in the case of acute liver failure, we have been timeously assisted by the MAC and the Minister of Health.

\section{Conclusion}

Current data suggests that the global burden of both acute and chronic liver diseases is expected to increase and we therefore estimate that the actual need for liver transplantation in children in Southern Africa will continue to grow as access to this life-saving treatment improves. ${ }^{15}$ Through international partnerships we have been able to successfully establish the first LDLT program in Sub-Saharan Africa and while this can to some extent satisfy the demand that deceased donor transplant currently is unable to supply for children awaiting liver transplantation, LDLT remains underused. Living donor liver transplantation requires substantial resources and for this and other programs to expand requires prioritization within the national healthcare agenda. ${ }^{16}$

\section{REFERENCES}

1. Spearman CWN, McCulloch MI. Challenges for paediatric transplantation in Africa. Pediatr Transplant. 2014;18:668-74.

2. Loveland J, Britz R, Joseph C, et al. Paediatric liver transplantation in Johannesburg revisited: 59 transplants and challenges met. S Afr Med J. 2014 Nov;104(11 Pt 2):799-802.
3. Dindo D, Demartines N, Clavien PA. Classification of surgical complications: a new proposal with evaluation in a cohort of 6336 patients and results of a survey. Ann Surg. 2004 Aug;240(2):205-13.

4. Mazariegos G, Shneider B, Burton B, et al. Liver transplantation for pediatric metabolic disease. Mol Genet Metab. 2014 Apr;111(4):418-27.

5. Patel N, Loveland J, Zuckerman M, et al. Heterozygote to homozygote related living donor liver transplantation in maple syrup urine disease: a case report. Pediatr Transplant. 2015 May;19(3):E62-5.

6. Fabian J, Crymble K. End-of-life care and organ donation in South Africa - it's time for national policy to lead the way. S Afr Med J. 2017 Jun 30;107(7):1947.

7. Ratner L, Sandoval P. When disaster strikes: Death of a living donor. Am J Transplant. 2010;10:2577-81.

8. Lauterio A, Di Sandro S, Gruttadauria S, et al. Donor safety in living donor liver donation: An Italian multicenter survey. Liver Transplantation. 2017;23(2):184-93.

9. Lee JG, Lee K-W, Kwon CHD, et al. Donor safety in living donor liver transplantation: The Korean Organ transplantation registry study. Liver Transplantation. 2017;23(8):999-1006.

10. Benzing C, Schmelzle M, Oellinger R, et al. Living-Donor Liver Transplant: An Analysis of Postoperative Outcome and HealthRelated Quality of Life in Liver Donors. Exp Clin Transplant. 2018 Oct;16(5):568-74.

11. Salvalaggio PR, Seda Neto J, Alves JA, et al. Consensus, Dilemmas, and Challenges in Living Donor Liver Transplantation in Latin America. Transplantation. 2016 Jun;100(6):1161-4.

12. Narasimhan G, Safwan M, Kota V, et al. Donor Outcomes in Living Donor Liver Transplantation-Analysis of 275 Donors From a Single Centre in India. Transplantation. 2016 Jun;100(6):1251-6.

13. Botha J, Conradie F, Etheredge $\mathrm{H}$, et al. Living donor liver transplant from an HIV-positive mother to her HIV-negative child: opening up new therapeutic options. AIDS. 2018 Oct 23;32(16):F13-F19.

14. Kasahara M, Sakamoto S, Fukuda A. Pediatric living-donor liver transplantation. Semin Pediatr Surg. 2017 Aug;26(4):22432.

15. Asrani SK, Devarbhavi H, Eaton J, Kamath PS. Burden of Liver Diseases in the World. J Hepatol. 2018 Sep 25 [Epub ahead of print]; pii: S0168-8278(18)32388-2. doi: 10.1016/j. jhep.2018.09.014

16. Song E, Fabian J, Boshoff PE, et al. Adult liver transplantation in Johannesburg, South Africa (2004 - 2016): Balancing good outcomes, constrained resources and limited donors. S Afr Med J. 2018;108(11):929-36. 